\title{
Perfil clínico e epidemiológico das alterações bucais em portadores do HIV
}

\author{
Clinical and epidemiological profile of oral changes in HIV patients \\ Perfil clínico y epidemiológico de las alteraciones bucales en portadores del VIH/SIDA \\ Júlio Leite de ARAÚJO-JÚNIOR ${ }^{1}$ \\ Ivo Cavalcante PITA-NETO ${ }^{2}$ \\ Julliana Cariry Palhano FREIRE ${ }^{3}$ \\ Eduardo DIAS-RIBEIRO ${ }^{4}$ \\ Residente em Cirurgia e Traumatologia Bucomaxilofacial, Hospital Universitário Lauro Wanderley, Universidade Federal da Paraíba-UFPB, \\ 58050.000 João Pessoa-PB, Brasil \\ ${ }^{2}$ Doutorado em Ciências da Saúde, Faculdade de Medicina do ABC Paulista-FMABC, 09060.870 Santo André-SP, Brasil \\ ${ }^{3}$ Doutoranda em Clínica Odontológica, Universidade Estadual da Paraíba-UEPB, 58429.500 Campina Grande, PB, Brasil \\ ${ }^{4}$ Professor do Programa de Residência em Cirurgia e Traumatologia Bucomaxilofacial,, Hospital Universitário Lauro Wanderley, \\ Universidade Federal da Paraíba-UFPB, 58050.000 João Pessoa-PB, Brasil
}

\begin{abstract}
Resumo
Introdução: Pacientes portadores do vírus da imunodeficiência humana apresentam susceptibilidade a desenvolver alterações bucais, em que estas estão intimamente relacionadas ao grau de carga viral e consequentemente a contagem de células linfocitárias. Objetivo: Traçar um perfil clínico-epidemiológico de alterações bucais em pacientes portadores do HIV. Material e Método: Realizou-se uma revisão onde foram analisados artigos científicos obtidos da base de dados PubMed. Foram incluídos os estudos do período de 2000 a 2017 , de acordo com os descritores: HIV; Linfócitos T CD4-positivos; Doenças da boca, bem como a associação dos termos. Totalizando-se 19 estudos para realização da pesquisa. As pesquisas que não se enquadraram a esses critérios foram excluídas. Os dados foram analisados a partir de estatística descritiva. Conclusão: Conclui-se que a cavidade oral é a área de maior frequência para manifestações do HIV/AIDS sendo a candidose, leucoplasia pilosa e as doenças periodontais as lesões mais ocorrentes em todo o mundo. Em relação ao sexo, conclui-se que a maior prevalência varia de acordo com a população estudada, sendo o sexo masculino o mais prevalente no Brasil, porém o sexo feminino quando afetado possuíam idade mais precoce.
\end{abstract}

Descritores: HIV; Linfócitos T CD4-Positivos; Doenças da Boca.

\section{Abstract}

Introduction: Patients with human immunodeficiency virus are susceptible to develop oral alterations, in which they are closely related to the viral load and consequently the lymphocyte count. Objective: To establish a clinical-epidemiological profile of oral alterations in patients with HIV. Material and Method: A review was carried out, where scientific articles were retrieved from the PubMed database. We included studies from the period 2000 to 2017, according to the descriptors: HIV; CD4-positive T lymphocytes; Diseases of the mouth, as well as the association of terms. A total of 19 studies were carried out to carry out the research. Searches that did not meet these criteria were excluded. Data were analyzed from descriptive statistics. Conclusion: It is concluded that the oral cavity is the area of greatest frequency for manifestations of HIV / AIDS being candidosis, hairy leukoplakia and periodontal diseases the most frequent lesions worldwide. Regarding sex, it was concluded that the highest prevalence varies according to the population studied, being the male sex the most prevalent in Brazil, but the female sex when affected had an earlier age.

Descriptors: HIV; CD4-Positive T-Lymphocytes; Mouth Diseases.

\section{Resumen}

Introducción: Los pacientes portadores del virus de la inmunodeficiencia humana son susceptibles a desarrollar alteraciones bucales, que están íntimamente relacionadas al grado de carga viral y al recuento de células linfocitarias. Objetivo: Trazar un perfil clínicoepidemiológico de alteraciones bucales en pacientes portadores del VIH. Material y Método: Se realizó una revisión bibliográfica, fueron analizados artículos científicos obtenidos de la base de datos PubMed. Se incluyeron los estudios del período 2000 a 2017, de acuerdo con los descriptores: VIH; Linfocitos T CD4-positivos; Enfermedades de la boca, así como la asociación de los términos. En un total de 19 estudios para la realización de la investigación. Las encuestas que no se relacionaron a estos criterios, se excluyeron. Los datos analizados a partir de estadística descriptiva. Conclusión: Se concluye que la cavidad oral es el área de mayor frecuencia para manifestaciones del VIH/SIDA siendo la candidiasis, leucoplasia pilosa y las enfermedades periodontal las lesiones más frecuentes en todo el mundo. En cuanto al sexo, se concluye que la mayor prevalencia varía de acuerdo con la población estudiada, siendo el sexo masculino el más prevalente en Brasil, pero el sexo femenino cuando afectado poseía edad más precoz.

Descriptores: VIH; Linfocitos T CD4-Positivos; Enfermedades de la boca

\section{INTRODUÇÃO}

De acordo com o relatório das nações unidas dois milhões de pessoas em todo o mundo foram infectados com HIV no ano de 2011, em comparação a este dado em 3,2 milhões de pessoas no ano de 2001. Embora a redução de infectados pelo HIV em todo o mundo tenha ocorrido, continua a ser um problema mundial de saúde pública, levando a mudanças demográficas profundas em países severamente afetados ${ }^{1,2}$.

A associação entre infectados pelo HIV e manifestações bucais tem sido considerada como uma importante característica desde o começo da epidemia em 1981 quando casos não explicados de sarcoma de Kaposi e de pneumonia por Pneumocystis carinni ocorriam em homossexuais masculinos em diversas cidades dos Estados Unidos, quando a síndrome da imunodeficiência adquirida (AIDS/SIDA) foi reconhecida oficialmente como doença pelos centros de controle de doenças e prevenção. Dessa forma as manifestações bucais passaram a ter valor diagnóstico e prognóstico importante. As manifestações bucais em pacientes infectados pelo HIV sugerem progressão do HIV para AIDS, também podendo ser mencionado que em pacientes sobre tratamento antirretroviral, manifestações bucais sugerem possível falhar do tratamento ${ }^{1,2}$.

Com o avanço da ciência e


desenvolvimento da terapia antirretroviral altamente ativa, o curso da doença AIDS tem sido modificado significativamente. Apesar do fato de quer a terapia antirretroviral tem sido altamente efetiva, a erradicação da doença não é possível, sendo assim o tratamento antirretroviral deduz a carga viral no plasma em uma extensão considerável, o qual resulta em uma elevação da contagem de células linfocitárias $\mathrm{TCD} 4^{3}$.

O presente estudo, por meio de revisão sistematizada, tem por objetivo traçar um perfil clínico e epidemiológico das alterações bucais em pacientes portadores do HIV buscando não só sua compreensão para melhor atendimento destes pacientes, como também determinar as principais manifestações bucais nos pacientes HIV/AIDS, promovendo segurança aos profissionais da equipe odontológico na incorporação dos procedimentos universais de biossegurança.

\section{MATERIAL E MÉTODO}

Uma estratégia de busca de artigos relacionados às alterações bucais em pacientes portadores do HIV foi realizada utilizando-se a base de dados PubMed por meio da combinação dos descritores: "HIV" and "CD4-Positive TLymphocytes" or "Mouth Diseases". Foi realizada também uma busca a partir da lista de referências dos artigos incluídos por meio das bases de dados. Como critérios de inclusão, foram levados em consideração: estudos realizados em humanos, adultos, estudos transversais tipo caso-controle, estudos clínicos randomizados controlados, relacionando diretamente HIV e doenças da boca, estudos escritos em inglês ou português e publicados na íntegra no período de 2000 a 2017. Foram excluídos artigos de revisão, estudos ecológicos, estudos de casos, estudos em animais, estudos sem grupo controle para comparação, estudos que apresentam apenas análise descritiva e estudos repetidos na base de dados. Os dados foram analisados a partir de estatística descritiva.

\section{RESULTADOS}

A estratégia de busca, depois de aplicados os critérios de inclusão/exclusão, resultou em um total de 19 artigos para compor esta revisão.

A AIDS pode apresentar as fases inicial, aguda, intermediária e avançada. A fase inicial pode ser assintomática enquanto a fase aguda surge num período que pode variar de duas semanas a seis meses. A fase intermediária é acompanhada por período de contenção imune do vírus, onde ocorrerá uma replicação do HIV latente de baixo nível. $\mathrm{Na}$ fase avançada o quadro é bastante variável com os sinais e sintomas das fases antecessoras podendo se manifestar, com um aumento do número de infecções oportunistas e final, às quais são acompanhadas de diversas repercussões clínicas ${ }^{4}$.
A contagem de linfócitos T CD4 na fase avançada é menor que $50 \mathrm{cel} / \mathrm{mm}^{3}$ e o tratamento se torna difícil pela dificuldade de se obter alguma resposta ${ }^{3}$. Manifestações bucais associadas à infecção por HIV deveram ser listadas baseando-se na intensidade com que se apresentam nesta associação dentro de três grupos. O grupo 1 é formado por lesões bucais associadas a infecção por HIV. O grupo 2 é formado por lesões de menor ocorrência. O grupo 3 é composto por lesões sem confirmação científica da associação a infecção pelo HIV (Tabela 1$)^{5}$.

A comunidade econômica europeia em 1986 em Copenhague discutiu os principais problemas bucais relacionados com a infecção pelo HIV, listando 30 doenças associadas. Posteriormente houve a necessidade de serem acrescentadas novas lesões que ocorriam na boca ou região submandibular. As lesões foram classificadas de acordo com sua natureza em lesões por infecção fúngicas, bacterianas e virais, com acréscimo de processos neoplásicos e de natureza desconhecida ${ }^{5,6}$.

As manifestações bucais associadas à infecção pelo vírus HIV foram classificadas como sendo de origem fúngicas, bacterianas, virais, neoplásicas e de origem desconhecida, tendo como base seu agente etiológico. As manifestações bucais mais frequentemente associadas ao HIV são: candidíase, leucoplasia pilosa, doença periodontal, ulcerações bucais, sarcoma de Kaposi, herpes, leucoplasia ${ }^{1,5}$.

Manifestações bucais ocorrem como resultado de doenças oportunistas em pacientes portadores do HIV ou em pacientes não tratados com terapia antiretroviral. Embora a ocorrência das manifestações bucais em portadores do HIV tenham diminuído após a introdução da terapia antiretroviral como sarcoma de Kaposi, leucoplasia pilosa bucal, doenças periodontais e grandes úlceras aftosas, a persistência da candidíase orofaríngea ainda tem sido observada ${ }^{5}$. Doenças bucais e doenças das glândulas salivares parecem ter aumentado entre os pacientes com terapia antirretroviral ${ }^{6}$. A literatura brasileira tem mostrado que o sexo masculino tem prevalecido quanto à infecção pelo HIV (Tabela 2$)^{5}$.

Tabela 1. Manifestações bucais baseadas na intensidade com que se apresentam nesta associação dentro de três grupos ${ }^{5}$.

\begin{tabular}{|c|l|}
\hline Grupo & \multicolumn{1}{|c|}{ Lesões } \\
\hline Grupo 1 & $\begin{array}{l}\text { Candidíase, leucoplasia pilosa, gengivite } \\
\text { úlcero-necrosante aguda (GUNA) e sarcoma de } \\
\text { Kaposi. }\end{array}$ \\
\hline Grupo 2 & $\begin{array}{l}\text { Vírus herpes, ulcerações atípicas, papiloma } \\
\text { vírus, doenças de glândulas salivares, varicela- } \\
\text { zoster e infecções virais por citomegalovírus. }\end{array}$ \\
\hline Grupo 3 & $\begin{array}{l}\text { Osteomielite, distúrbios neurológicos, sinusite e } \\
\text { carcinoma epidermóide. }\end{array}$ \\
\hline
\end{tabular}


Tabela 2. Prevalência de manifestações bucais quanto a variável sexo ${ }^{5}$.

\begin{tabular}{l|c|c}
\hline \multirow{2}{*}{ Manifestações Bucais } & \multicolumn{2}{|c}{ Sexo } \\
\cline { 2 - 3 } & Masculino & Feminino \\
\hline Candidíase & 72 & 21 \\
\hline Leucoplasia Pilosa & 5 & 1 \\
\hline Sarcoma de Kapose & 5 & - \\
\hline Herpes Simpes & 4 & 2 \\
\hline GUNA & 4 & - \\
\hline Gengivite & 30 & 11 \\
\hline Periodontite & 29 & 8 \\
\hline Úlceras Tuberculoide & 1 & - \\
\hline Nenhuma & 4 & 4 \\
\hline Total & 154 & 47 \\
\hline
\end{tabular}

A literatura tem classificado as manifestações bucais em portadores do HIV de acordo com a intensidade com que ocorrem na cavidade bucal, local anatômico mais acometido, apresentação clínica, assim como a associação entre a contagem de linfócitos T CD4 $4^{1,7,8}$.

Pesquisadores tem observado uma redução da prevalência de manifestações bucais por infecções oportunistas com início da terapia antirretroviral combinada $^{9,10}$. Esse dado vantajoso tem ocorrido devido a uma recuperação parcial das funções do sistema imunológico após supressão da viremia e redução da destruição celular causada pelo HIV, além de um possível efeito antiapoptose de algumas drogas ${ }^{9}$. A terapia antirretroviral acarreta diminuição da carga viral do HIV, com consequente aumento dos linfócitos T CD4, resultando em menor frequência das lesões oportunistas ${ }^{9}$. Falhas na terapêutica podem ocorrer, sendo indicadas pelo aparecimento das lesões bucais na vigência do tratamento ${ }^{5}$.

\section{DISCUSSÃO}

Foram coletados dados de diversas pesquisas relevantes, as quais variaram muito devido a fatores tais como estágio de AIDS e tipo de regime da terapia retroviral. Outros fatores importantes como a idade, competência do sistema imunológico, hábito de fumar, níveis de higiene bucal nem sempre são levados em consideração em muitas dessas pesquisas $^{8,11}$.

Por meio de estudos realizados em todo o mundo, observa-se que após a introdução da terapia antirretroviral houve um declínio no número de internações hospitalares sem, contudo, levar à queda do número de infecções oportunistas e manifestações bucais $^{1,12}$.

Com relação às manifestações bucais houve uma diminuição significativa na prevalência de $45 \%$ a $85 \%$ até cerca de $32 \%$ a $46 \%$ após a introdução da terapia antirretroviral ${ }^{1,5}$. No entanto, estudo realizado por Aquino-Garcia et al. ${ }^{14}$ uma taxa mais baixa de manifestações orais pode ser observada em uma prevalência de $32 \%$, observada em pacientes sobre terapia antiretroviral ${ }^{3}$. De um modo geral, no Brasil foram registradas taxas significativamente mais baixas de manifestações bucais ${ }^{13,14}$.

A contagem de linfócitos T CD4 e da viremia plasmática (quantidade de partículas virais) são exames fundamentais. Elas fornecem dados quanto à forma de progressão da doença, o grau de imunodeficiência e a velocidade de destruição do sistema imunológico, avaliando também o risco de desenvolvimento de doenças oportunistas. É através deste exame que se planeja e institui a profilaxia medicamentosa para infecções oportunistas mais frequentes ${ }^{7}$.

Tratando-se de pacientes HIV positivo e lesões bucais, não só linfócitos T CD4 abaixo de 200 cél $/ \mathrm{mm}^{3}$, como também uma carga viral elevada, higiene bucal precária, uso de tabaco e xerostomia podem contribuir para o desenvolvimento precoce dessas lesões bucais ${ }^{11,15}$.

A contagem de células T CD4 acima de $600 \mathrm{cel} / \mathrm{mm}^{3}$ é tida como normal, e a supressão imune inicial é definida como sendo abaixo de $500 \mathrm{cel} / \mathrm{mm}^{3}$. A literatura chama atenção para casos de severa imunodepressão onde se tem uma contagem de células T CD4 abaixo de $200 \mathrm{cel} / \mathrm{mm}^{3}$, tendo perda anual de 60 a $80 \mathrm{cel} / \mathrm{mm}^{3}$ podendo apresentar variações de indivíduo para indivíduo ${ }^{9}$. Com a diminuição na contagem de linfócitos $\mathrm{T}$ CD4, principalmente em indivíduos com níveis inferiores a $200 \mathrm{cel} / \mathrm{mm}^{3}$, o aparecimento de lesões bucais associadas ao HIV se mostra aumentado ${ }^{7}$.

Quanto à prevalência de lesões bucais relacionadas com o HIV observou-se prevalência significativamente maior nas mulheres do que em homens, sendo a candidíase bucal a lesão mais comumente observada nesse grupo ${ }^{16,17}$. No que diz respeito à leucoplasia pilosa aparece em maior número nos homens fumantes, mostrando que as manifestações bucais variam em prevalência em se tratando de sexo, sugerindo as diferenças no estilo de vida desses dois grupos ${ }^{16,17}$.

Estudos conduzidos por Braz-Silva et al. $^{8} \mathrm{e}$ Lins et al. ${ }^{17}$ mostram resultados divergentes daqueles obtidos por Ravi e Rao ${ }^{1}$ e Davis et al. ${ }^{6}$ em relação à influência da variável sexo com maior prevalência das lesões bucais no gênero masculino.

As condições bucais associadas ao HIV/AIDS são representadas pela candidíase bucal, leucoplasia pilosa, sarcoma de Kaposi, eritema gengival linear, periodontite necrosante ulcerativa e úlcera aftosa. Outras lesões menos frequente relatadas em alguns artigos são infecção humana do papillomavirus, hiperpigmentação, fibrose submucosa oral, xerostomia, leucoplasia, herpes zoster, linfoma não-Hodgkin, histoplasmose, carcinoma, penicilliosis marneffei, queilite esfoliativa, doença das glândulas salivares, molusco 
contagioso perioral, infecções por staphylococcus aureus e petéquias ${ }^{1,2,18,19}$.

\section{CONCLUSÃO}

Conclui-se que a cavidade oral é a área de maior frequência para manifestações do HIV/AIDS sendo a candidose, leucoplasia pilosa e as doenças periodontais as lesões mais ocorrentes. Em relação ao sexo conclui-se que a maior prevalência varia de acordo com a população estudada, sendo o sexo masculino o mais prevalente no Brasil, porém o sexo feminino quando afetado possuíam idade mais precoce.

\section{REFERÊCIAS}

1. Ravi JR, Rao TR. Estimation of prevalence of periodontal disease and oral lesions and their relation to $\mathrm{CD} 4$ counts in HIV seropositive patients on antiretroviral therapy regimen reporting at District General Hospital, Raichur. J Indian Soc Periodontol. 2015; 19(4):435-439.

2. Pakfetrat A, Falaki F, Delavarian Z, Dalirsani Z, Sanatkhani M, Zabihi Marani M. Oral manifestations of human immunodeficiency virus-infected patients. Iran J Otorhinolaryngol. 2015; 27(78):43-54.

3. Berberi A, Aoun G. Oral lesions associated with human immunodeficiency virus in 75 adult patients: a clinical study. J Korean Assoc Oral Maxillofac Surg. 2017; 43(6):388-94.

4. Patton LL. Oral lesions associated with human immunodeficiency virus disease. Dent Clin North Am. 2013; 57(4):673-98.

5. Frimpong P, Amponsah EK, Abebrese J, Kim SM. Oral manifestations and their correlation to baseline CD4 count of HIV/AIDS patients in Ghana. J Korean Assoc Oral Maxillofac Surg. 2017; 43(1):29-36.

6. Davis G, Perks A, Liyanage P, Staines K. Oral hairy leukoplakia arising in a patient with hairy cell leukaemia: the first reported case. BMJ Case Rep. 2017. pii:bcr-2016-218663.

7. Berberi A, Noujeim Z. Epidemiology and relationships between $\mathrm{CD} 4+$ counts and oral lesions among 50 patients infected with human immunodeficiency virus. J Int Oral Health. 2015; 7(1):18-21.

8. Braz-Silva PH, Schussel JL, López Ortega $\mathrm{K}$, Gallottini M. Oral lesions as an important marker for HIV progression. Dermatol Online J. 2017; 23(9):13030/qt9t26m7n3.

9. Eyer-Silva WA, Soares PEMA, Azevedo MCVM, Silva GARD, Signorini DJHP, NevesMotta $\mathrm{R}$ et al. An unusual case of bacillary angiomatosis in the oral cavity of an AIDS patient who had no concomitant tegumentary lesions - case report and review. Rev Inst Med Trop Sao Paulo. 2017; 59:e59.
10. Al-Kzayer LF, Keizer P, Abdulraheem FT, Sano K, Kamata M, Sakashita K et al. Rapidly progressive Kaposi's Sarcoma in an Iraqi boy received Valproic acid: a case report and review of literature. BMC Pediatr. 2016; 16:111.

11. Arinze F,Shaver A, Raffanti S. Surgical excision for recurrent herpes simplex virus 2 (HSV-2) anogenital infection in a patient with human immunodeficiency virus (HIV). Infection. 2017; 45(5):705-7.

12. Rossi A, Ferreira L, Cuevas-Nunez M, Wright JM, De-Paula AMB, Basile JR et al. Angiopoietin-2 is expressed in oral Kaposi's sarcoma. J Oral Pathol Med. 2017; 46(10):1011-14.

13. Ferreira S, Noce C, Júnior AS, Gonçalves L, Torres S, Meeks V et al. Prevalence of oral manifestations of HIV infection in Rio de Janeiro, Brazil from 1988 to 2004. AIDS Patient Care STDS. 2007; 21(10):724-31.

14. Aquino-García SI, Rivas MA, CeballosSalobreña A, Acosta-Gio AE, Gaitán-Cepeda LA. Short communication: oral lesions in HIV/AIDS patients undergoing HAART including efavirenz. AIDS Res Hum Retroviruses. 2008; 24(6):815-20.

15. Arutyunyan S, Uhde M. Benign lymphoepithelial lesion of the parotid gland in the Setting of HAART. J Int Assoc Provid AIDS Care. 2017; 16(2):120-24.

16. Vale DAD, Rogado CM, Carvalho DLC, Trierveiler M, Ortega KL. Oral plasmablastic lymphoma as the first manifestation of AIDS. An Bras Dermatol. 2017; 92(5 Suppl 1):110-12.

17. Lins L, Farias É, Brites-Alves C, Torres A, Netto EM, Brites C. Increased expression of CD38 and HLADR in HIV-infected patients with oral lesion. J Med Virol. 2017; 89(10):1782-87.

18. d'Ettorre G, Ceccarelli G, Giustini N, Serafino S, Calantone N, De Girolamo G et al. Probiotics Reduce Inflammation in Antiretroviral Treated, HIV-Infected Individuals: Results of the "Probio-HIV" Clinical Trial. PLoS One. 2015; 10(9):e0137200.

19. Konaté A, Barro-Kiki PCM, Kassi KF, Angora $\mathrm{KE}$, Vanga-Bosson $\mathrm{H}$, Djohan $\mathrm{V}$ et al. Oropharyngeal candidiasis prevalence among HIV-infected patients at the teaching hospital of Treichville (Abidjan, Côte d'Ivoire). J Mycol Med. 2017; 27(4):549-53. 


\section{CONFLITO DE INTERESSES}

Os autores declaram não haver conflitos de interesse.

\section{AUTOR PARA CORRESPONDENCIA}

\section{Júlio Leite de Araújo Júnior}

juniorleitearaujo@hotmail.com

Submetido em 02/04/2018

Aceito em 06/06/2018 